\title{
Potencial de las microempresas en el sector gastronómico en el municipio de Tlahuelilpan en el estado de Hidalgo.
}

\author{
Potential of micro-enterprises in the gastronomic sector in the municipality of Tlahuelilpan in \\ the state of Hidalgo. \\ Edgar Ubaldo Paredes Sánchez , Ángel Tadeo Velásquez Monroy ${ }^{b}$, Aarón Vargas Aguilar ${ }^{\text {, }}$ \\ Irma Isabel de León Vázquez, ${ }^{d}$, Ivette Flores Jiménez ${ }^{e}$
}

\begin{abstract}
:
This work shows the potential of micro-enterprises in the municipality of Tlahuelilpan in the State or Hidalgo, having tha main objective of describing the future of small fast food businesses, through the analysis of resistance to change to guide businesses with improvements that will allow them to stay in the future. Just as we describe the small fast food businesses in the center of the municipality to see the reasons that help them improve their internal administration and stay current in the long term. The results are presented, the reasons why they are valid and a guide that serves to know how to make changes to the business and achieve its stability in the market.
\end{abstract}

Keywords:

Resistance, change, business, markets, administration.

\section{Resumen:}

Este trabajo muestra el potencial de las microempresas del municipio de Tlahuelilpan en el Estado de Hidalgo, al tener como principal objetivo el describir el futuro de los pequeños negocios de comida rápida, a través del análisis de la resistencia al cambio para guiar a los negocios con mejoras que les permita permanecer en un futuro. Así como describe los pequeños negocios de comida rápida en el centro del municipio para ver las razones que les consienta mejorar su administración interna y seguir actuales a largo plazo. Se presentan los resultados, las razones por las cuales están vigentes y una guía que servirá para saber hacer cambios al negocio y lograr su estabilidad en el mercado.

Palabras Clave:

Resistencia al cambio, negocios, mercado, administración.

\section{Introducción}

El presente documento tiene como objetivo evaluar el potencial de las microempresas del sector gastronómico

\footnotetext{
a Autor de Correspondencia, Universidad Autónoma del Estado de Hidalgo, Escuela Superior de Tlahuelilpan, Alumno de la Licenciatura en Administración, ORCID: https://orcid.org/0000-0001-8776-7439,Email: pa405014@uaeh.edu.mx

b Universidad Autónoma del Estado de Hidalgo, Escuela Superior de Tlahuelilpan, Alumno de la Licenciatura en Administración, ORCID: https://orcid.org/0000-0002-9312-8470, Email: ve414460@uaeh.edu.mx

c Universidad Autónoma del Estado de Hidalgo, Escuela Superior de Tlahuelilpan, Alumno de la Licenciatura en Administración, ORCID: https://orcid.org/0000-0001-5321-5505, Email: va414069@uaeh.edu.mx

d Universidad Autónoma del Estado de Hidalgo, Escuela Superior de Tlahuelilpan, Profesor por Asignatura de la Licenciatura en Administración, ORCID: https://orcid.org/0000-0001-9197-0050,Email: irmalv@ uaeh.edu.mx

e Universidad Autónoma del Estado de Hidalgo, Escuela Superior de Tlahuelilpan, Profesor de Tiempo Completo de la Licenciatura en Administración, ORCID: https://orcid.org/0000-0002-9591-4416,Email: ivette@uaeh.edu.mx
} 
en el centro del municipio de Tlahuelilpan en el Estado Hidalgo, mediante una encuesta para determinar cuál es su estado actual y cómo influye la publicidad que se mantiene en constante cambio para atraer más clientela. Por ello, es importante que los negocios sepan cómo hacer cambios en su entorno para mantenerse vigentes en el mercado, así como observar las áreas de oportunidad que tienen aquellos negocios que se quedan estancados y la propuesta de una guia para mejorar el negocio.

La decision de iniciar un negocio de comida rápida como los que ofrecen desayunos, antojitos o comidas, tiene que tener como propósito el mantenerse vigentes en el mercado, pero la supervivencia de éstos dependen en gran medida del dueño, sin embargo, para que su negocio o establecimiento quede expuesto a entrar en quiebra no es necesario hacer grandes cantidades de inversión que muchos hacen pero que no ven retribuido con la generación ingresos; ésto se puede deber a la mala publicidad del negocio que no es lo suficientemente llamativa o al servicio que se brinda al cliente.

Los clientes o usuarios del servicio no solo prefieren un lugar por la comida, si no por la manera en que son atiendidos, por ellos es importante que los que atienden esten en la mejor disposion de dar un buen servicio, otra razón que debe revisar el establecimiento es la forma en que preparan el producto ya que aveces los comensales acuden al lugar por el zasón que se tiene, ésta es una forma de poder posicionarse y con ello tener la posibilidad de recuperar lo invertido con el aumento de las ventas, generando ganancias.

En México se consume mucho la comida rápida, lo que permite que los desayunos o comedores sean populares por la forma de preparar los alumentos, pero la competencia que se presenta en esta área, obliga a las pequeños establecimientos a considerar la posibilidad de hacer cambios de manera constante para mantenerse vigente en el mercado.

También se sugerible que tengan un enfoque en la vigencia que tienen algunos productos y que es necesario a veces meter o sustitu ir agunos alimentos.

Las ganacias que obtienen algunos de estos establecimientos son lo suficiente para el constante mejoramiento de éstos, pero aveces no saben que hacer para atraer más clientes y podría considerarse el introducir nuevos alimentos, o invertirle a su publicidad o expandirse en algunos casos para poder tener más espacio que le sea lo suficiente para poder tener mayor cantidad de comensales.

El centro del municipio de Tlahuelilpan algunos de los estudiantes carecen de tiempo para preparar su propia comida o lounch y buscan una variedad de comida que va desde antojitos, desayuos o comidas en forma y en algunas ocasiones ya se preocupan que éstas sean cada vez más nutritivas para el bienestar de ellos mismos.

Por otro lado, estos negocios a pesar de ser pequeños cuentan con personal que su única forma para obtener un ingreso es através de dar este servicio de comida rápida conviertiéndose en generadores de empleo y por ello es importante apoyarlos indicándoles que se debe contar con objetivos, metas e ideas para de esta manera ataer más clientes, posicionarse en el marcado y minimizar el riesgo de desaparecer en poco tiempo.

\section{Reporte de práctica}

La gastronomía de Tlahuelilpan es un sector que esta respaldada por la ubicación geografica que tienen los negocios de comida rápida ya que sus clientes no solo son estudiantes y docentes de la universidad o escuelas establecidas en el municipio, también hay gente que viene de otros municipios o estados.

Si se observa el tráfico de personas que transitan por el centro del municipio de Tlahuelilpan, se puede considerar que se tiene un gran potencial de consumidores, por lo que los negocios deberían considerar el uso de estrategias que les permitan atraer a clientes potenciales y para ello tendrían los dueños de negocios que entender la importancia de los cambios que puedan realizar en el establecimiento invertiendo en mejoras de imagen, alternativas de diversos tipos de comida que puedan ofrecer o también en la manera en que atiende a los clientes.

Considerando el movimiento migratorio que tiene el municipio por parte de la universidad y de otras comunidadesque llegan a pasarpor él, genera una fuerte entrada de dinero, pero también se indentifican oportunidades que se tienen para ganar clientes, el cambio en un negocio no es siempre fácil, pero es muy importante para su permanencia en el mercado, el potencial que tienen los negocios, como se comentó anteriormente, en su mayoría es gracias a que los clientes los recomiendan ya sea por sus productos, la rapidez y calidez del servicio o por su ubicación, por ello los micronegocios de comida rápida deben sar administrados para que en un futuro sigan no como se encuentran actualmente, sino que se observe un crecimiento.

Para mejorar un negocio es necesario convencer a los dueños de invertir en marketing, mostrándole las estrategias que puede seguir, para logralo se debe contar como objetivo el aumentar las ventas para tener una mayor utilidad, contratar personal que labore considerando lo importante de atender al cliente (porque 
un cliente satisfecho generará mayores ventas), y que la ubicación de éstos es fundamental.

Por ello, es necesario saber los recusos con los que cuentan y llevarunabuena administración de los mismos, conocer la capacidad que tiene en cuanto al personal, productividad y la ventaja competiva con la cuentan para sobresalir de los otros negocios, conocer el escenario en que se desenvulve e identificar cuáles son sus áreas de oportunidad y evitar posibles fallas.

No se puede dejar la lado la importancia de observar las condiciones de higiene y notar si los clientes estan a gusto con el servicio que se les brinda, también percibir la calidad de la comida, todo influye en el precio; por lo tanto, si se mejoran estos aspectos antes mencionados se puede mejorar el precio y con ello generar mayor utilidad.

Para que el dueño de un micronegocio de comida rápida invierta en su negocio es porque asegura la generación de utilidades que les sea de gran ayuda, sin embargo, muchos de ellos ésperan estos resultados, pero no saber como administrar sus negocios. Desconocen cómo llevar un control de los recursos que ocupan o cuánto es lo que gastan lo que les impide deteminar sus ganancia.

Con base en todo o anterior, se determinó como objetivo general el describir el futuro de los pequeños negocios de comida rápida del centro del municipio de Tlahuelilpan en el Estado de Hidalgo, a través del análisis de la resitencia al cambio de los dueños, para proponer una guia a los negocios con mejoras que les permita permanecer en el futuro.

Siendo en el trabajo sus objetivo específicos:

1. Indentificar los negocios de la zona centro del municipio de Tlahuelilpan, en el estado de Hidalgo, a través de un censo para realizarles una encuesta.

2. Indentificar problemas que puedan tener los negocios con los resultados de la encuesta para establecer su situación conforme a la "Resistencia al cambio".

3. Establecer una guía con indicaciones básicas de cambios para permanecer en el mercado futuro.

Cómo marco referencial, se realizarón diversas lecturas a documentos que permitieron identificar los siguientes puntos: los negocios de comida rápidas tienen un gran número de clientes que los respalda para seguir vigentes en el mercado, esto se debe a ubicación, la pregunta es cuántos de estos pequeños negocios de comida permanecerá por largo tiempo.

La encuesta será una manera de conocer cómo estan conformadas las condciones de trabajo, ya que al tener una clientela que satisfacer se pueden identificar los factores de permanencia como son el sabor, la publicidad $y$ el servicio que ofrece con la meta de poder mantenerse vigentes en el mercado. Existen micronegocios que tiene una variedad de alimentos para desayunar, comer, o incluso cenar y que pueden consumir los estudiantes

Un negocio de comida rápida es un establecimiento donde se brinda un servicio a los comensales que estan buscando satisfacer una necesidad primaria del hombre - comer.

Una administración adecuado en los negocios de comida tiene que ver con el control de los gastos que tiene como son el gas, materia prima (viveres para la preparación), luz, agua, renta del lugar, personal que atienda, etc. por mencionar algunos. Para mantenerse vigente es necesario el contar con personal que se sepa mover en la areas.

La visión y misión forman parte de la planeación estratégica de un negocio, porque permite saber cómo y qué es lo que tienen que hacer, por ello es importante que se tengan establecidos por parte del dueño y que sus empleados las conozcan, además de estar abierto para adaptarse a los cambios necesarios en lo que corresponde a las estrategias de marketing, como es la publicidad, la imagen.

Si se lográ lo anterior de manera adecuada el dueño puede pensar en abrir otros establecimientos permita al negocio crecer y tener mas ingresos que no solo se generen en el centro de Tlahuelilpan Hidalgo, si no en otros municipios aledaños como Tula, Tlaxcoapan, Mixquiaula o Tezontepec.

Algunos negocios se han adaptado a cambios de manera inconciente, pero que les ha permitido crecer $y$ permanecer en el mercado, como suele ser: realizar cambios en el menú, diferentes tipos de alimentos como refrigerios (lo que es muy práctico para quienes cuentan con poco tiempo), colocar menús por temporadas.

Siempre a los clientes les interesa mucho la calidez con que son atendidos, o la rapidez en que sirven los alimentos (sobre todo en horas pico la preparación es fundamental), con ésto el negocio se puede volver más popular.

Por otro lado el ámbiente de trabajo en un negocio de comida puede ser un factor importante para su permanencia o quiebra; mucho de un buen ambien laboral tiene que ver con situaciones económicas generando rose entre los empleados y eso afecte el ambiente de trabajo.

La capacitación en este tipo de negocios no es considerada importante, pero es fundamental para que el personal cuente con la disposición de hacer lo que 
aprendio, de tal manera que no por un empleado se pare la productividad que se tiene en el mismo negocio o se dé un mal servicio.

Otro aspecto es la publicidad su impacto tiene que verse reflejado al atraer nuevos clientes al negocio, el servicio es una manera de ganar clientes ya que si el establecimiento se vuelve popular y la gente comienza a hablar de él, puede generar nuevas relaciones con los clientes que lo empiezen a recomendar, la ubicación como se mencionó anteriormente es fundamental para ofrecer más debido a la cercania. Esto es lo que le suceda a los negicios de comida en el centro del municipio de Tlaheulipan por su cercania con la universidad e instituciones educativas.

\section{Marco referencial}

De acuerdo a lo comentado por David Polo Mota en el 2017 en ACADEM - Escuela Latinoamericana de Management y Marketing. Las razones por las cuales se acercan clientes a un establecimiento de comida rápida son:

1. Rapidez

2. Acceso fácil

3. Precios moderados

4. Cuenta con estándares de procesos, platillos, administración y operación

Los establecimientos de comida rápida del centro del municipio de Tlahueilpan tienen la ventaja de que los alimentos pueden ser ingeridos por individuos de cualquier edad, son fáciles de preparar, algunos son saludables, resultan ser practicas cuando no se dispone de poco tiempo para comer, son accesibles al bolsillo para quienes no se cuenta con suficiente dinero para poder comer en un restaurante o pedir para llevar.

Revisando material bibliográfico se considera que las Estrategias de Marketing (Aim16) que pueden aplicar en:

1. Definir el proceso de elaboracion de la comida.

2. Gestionar quejas.

3. Capacitar al personal.

4. Crear un ambiente saludable entre los empleados.

Para lograr el objetivo planteado al inicio del documento se consideró dos tipos de respuestas que pemitiran ver cómo se encuentran los negocios de comida en lo que respecta al personal, producto, misión, visión y cómo se encuentran conforme a la aceptación al cambio.

\section{Abiertas}

- Las preguntas abiertas tratan de que haiga una respuesta donde el entrevistado, trate de expresarse con mas libertad conforme a lo que' piensa sobre la pregunta.
Cerradas (opcion multiple)

-Son preguntas en donde nadamas se tiene que eligir ente ciertas cantidades de respuestas que se hace al entrevistado.
El método de análisis se estableció con base a la siguiente clasificación:

Vertical o estático.- Permite cuantificar las entrevistas.

Horizontal o dinámico.- Compara la situación de los negocios para determinarpor qué uno es más exitoso que otro.

Histórico.- Permite ver cuál fue el impacto de la llegada de la universidad para los negocios de comida rápida.

Proyectado o estimado.- Permite guiar a los pequeños negocios de comida rápida para que tengan una buena administración.

Inductivo.- Sugiere una serie de pasos para una investigación sobre el mercado y el comportamiento del cliente.

Ahora bien, en lo que corresponde al comportamiento del cliente, esto se refiere al estudio psicológico que se tiene para determinar la mentalidad que tiene para consumir.

A continuacion se muestra los factores que influyen en el comportamiento del consumidor. (Aim16)
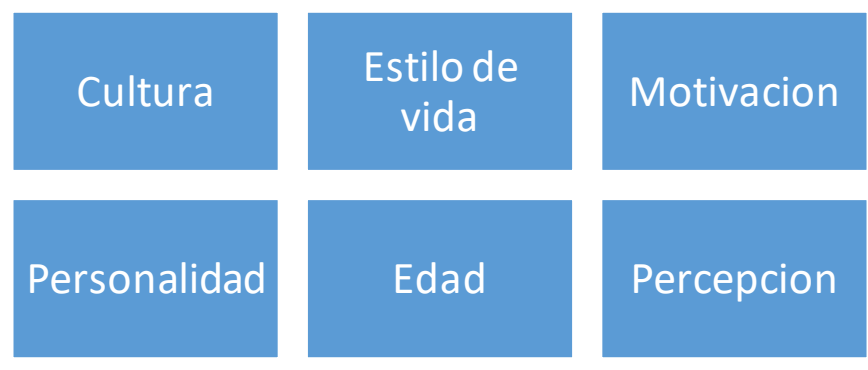

Percepcion

\section{Método}

Se llevó a cabo la encuesta y los resultados obtenidos con respecto a cómo se encuentran los pequeños negocios de comida rápida en el centro del municipio de Tlahuelilpan y determinar las áreas de oportunidad 
Se obtu vieron datos cuantitativos que permitieron realizar el análisis, las encuestas que se aplicaron permitierón proponer soluciones a la administracion del establecimieto, beneficios a los negocios y mejor conocimiento de que tienen que hacer para atraer mas clientes y con ello generar mejor utilidad.

El proceso fue el siguiente:

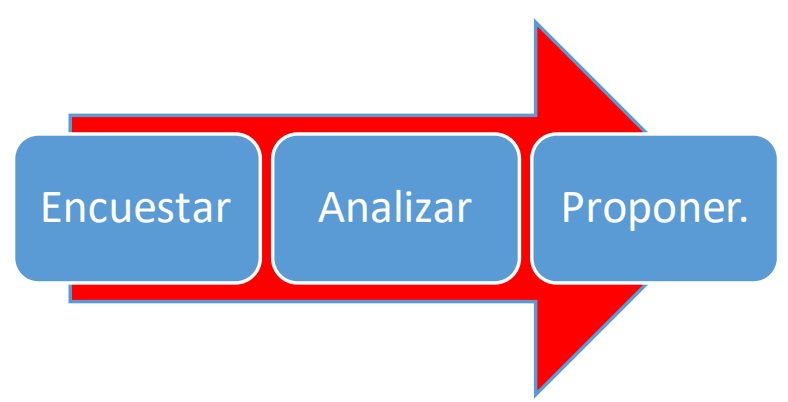

Se utilizó una metodologia mixta porque se hace una combinacion de datos cuantitivos y datos cualitativos, lo cual tiene una combianacion que permite analizar la situacion de cada puesto.

Las dos metologias se combinaron en todo el proceso de investigacion, ambas tipos de preguntas son necesarios para poder analizar lo que comentan de su administración cada puesto de comida rápida. Esto hace que la investigación se enfoque en puntos específicos que son fundamentales para que un negocio sepa cómo mantenerse vigente en el mercado.

El método inductivo.- es para poder sacar conclusiones generales, porque es uno de los métodos de investigacion más usados, ésto permite ir de lo general a lo particular, se basa en la observación y estudio. Este método va servir para indentificar algunos puntos en los que estan ejecutando los negocios que les permitira sacar una conclusión y poder proponer una solución.

El método deductivo.- se basa en el pensamiento o razonamiento; para poder extraer conclusiones lógicas a partir de proposiciones, es decir se basa en principios y hechos concretos. Lo que permite en el ensayo llegar a conclusiones lógicas de cómo pien sa el consumidor sobre los establecimientos o puestos para que se le pueda mejorar.

Los métodos pemitierón:

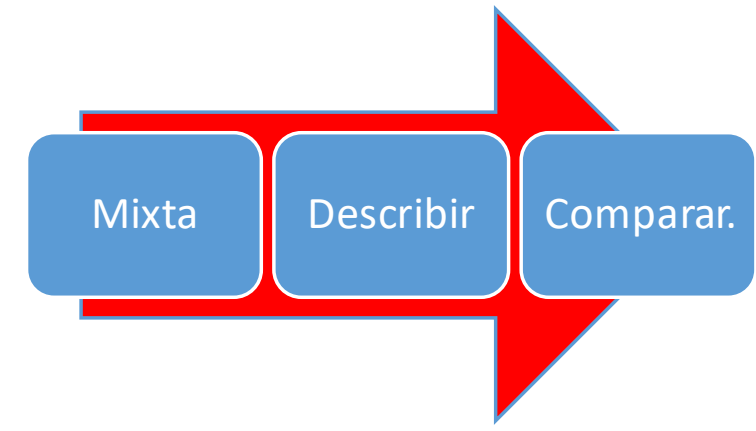

La población y muestra que se utilizó en el trabajo de investigacion esta representada en la imagen topográfica, en donde se puede observar los establecimientos de comida rápida que se encuentran en el centro del municipio de Tlahuelilpan en el Estado de Hidalgo. Los puntos rojos hacen referencia a las microempresas de comida que se les realizó encuesta con el fin de recabar información.

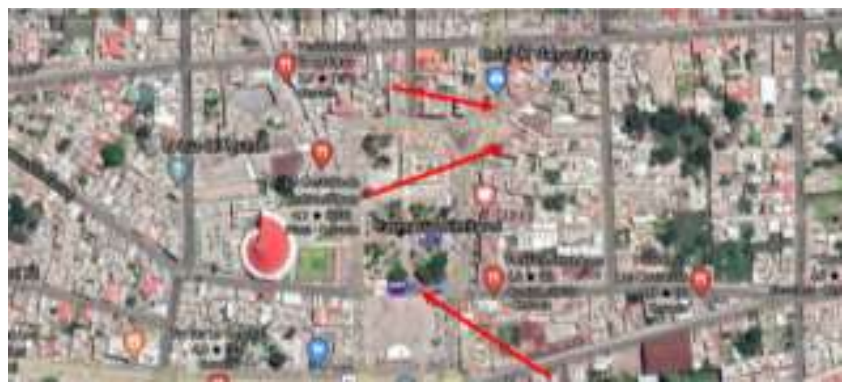

Estos establecimientos fueron "La castañeda", "La cocina el Peque", "Las hamburguesas", "tortas la central", "corredor de puesto de comida frente al teatro al aire libre", "desayunos contra esquina de la universidad".

Los puestos se encuentra cerca de la escuela primaria Melchor Ocampo, cerca de la Universidad y de la Preparatoria Sor Juana Inés de la Cruz.

\section{Resultados}

De las encuestas que se realizarón de presentan solo algunos de los resultados grafícados que se considerán relevantes para el reporte de investigación: 


\section{LOS CAMBIOS QUE HAN HECHO PARA MANTENERSE VIGENTE SE HAN HECHO EN FUNCIÓNDE:}

Estudiantes $\square$ Civil $₫$ Otra (trabajadores, chofers)

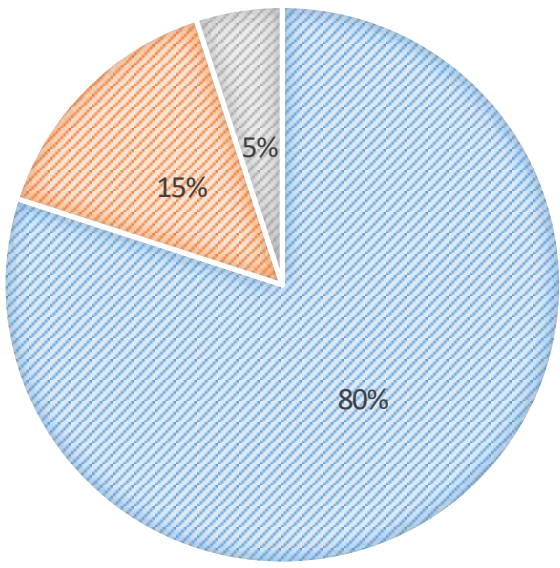

\section{CON QUIÉN ESPERA IMPACTE SU PUBLICIDAD}

Estudiantes $\backsim$ Civil $=$ Otra (trabajadores, chofers)

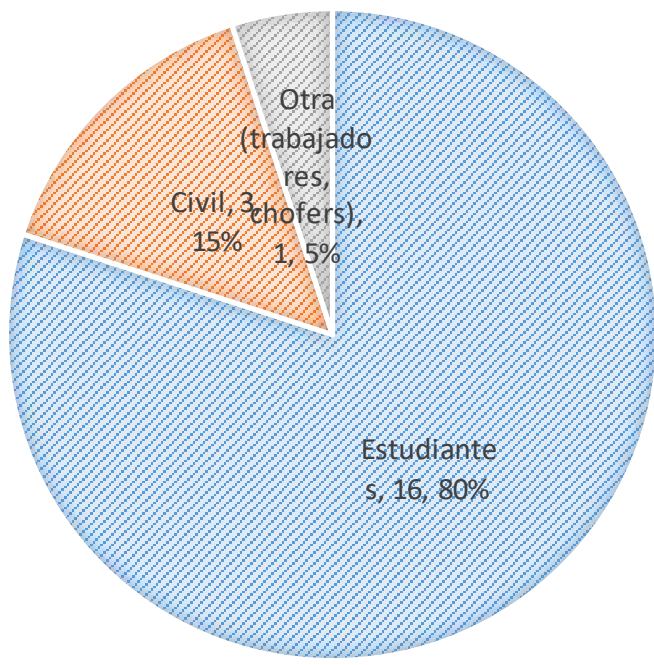

\section{QUÉ CLIENTES SE SIENTES MOTIVADOS CON SUS SERVICIOS}

astudiantes $\square$ Civil $\square$ Otra (trabajadores, chofers)
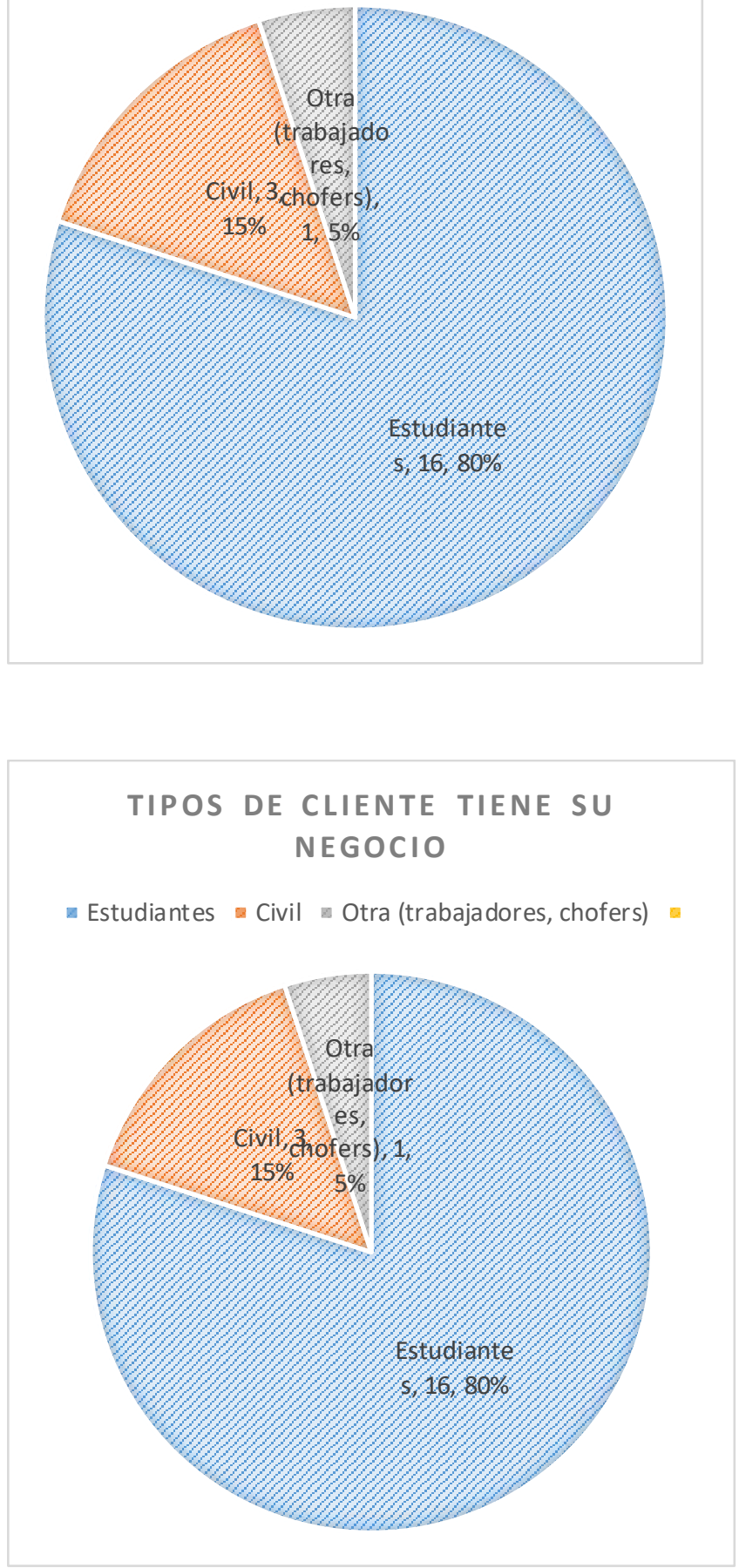


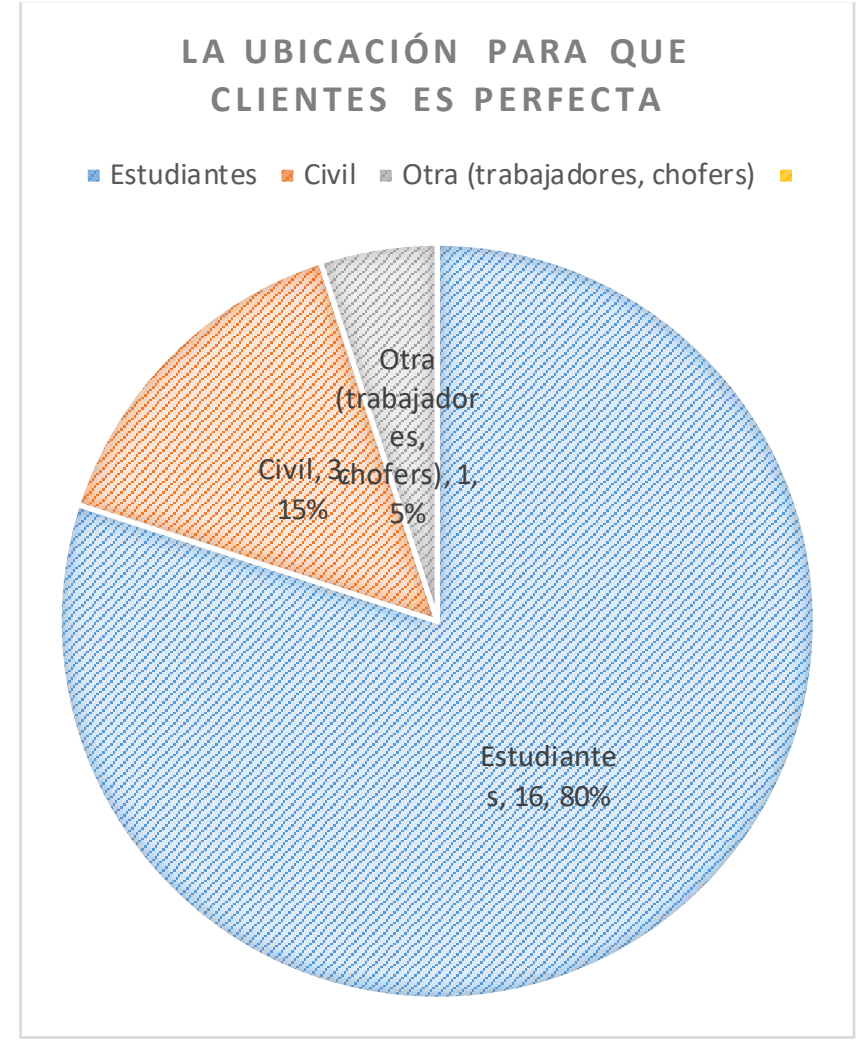

\section{Discusión}

En lo que corresponde a las encuestas que se aplicarón se tomarón 20 negocios entre desayunos, comedores, locales y puestos de comida rápida. En lo que se pudo observar lo siguiente:

\section{Aceptación de los cambios que han tenido:}

Varios negocios han perdido clientes, otros no era lo que ellos esperaban, pero en lo general los puestos han sabido aceptar los cambios y han cambiado ellos también para poder seguir brindando un servicio.

2. Identifican la visión y misión del negocio:

Varios de los establecimientos el dueño ten ía que estar al pendiente de las ganancias pero no sabía administrar el dinero e invertirlo en el negocio, otras veces el dueño se encuentra satisfecho con lo que produce sin darse cuenta que no logra cubrir sus gastos y que puede estar a punto de irse a la bancarota por una mala administración.

3.Cómo mantenerse vigentes en el mercado:

Varios de los establecimientos de comida rápida se vieron en la necesidad de incorporar nuevo producto o incluso mejorar el precio, otros tuvieron que mejorar el lugar de servicio debido a que los clientes buscan mayor comodidad.
Factor de análisis

Negocios

\begin{tabular}{l|l|}
\hline Realizarón cambios en su publicidad. & 5 \\
\hline $\begin{array}{l}\text { Realizarón cambios en sus productos. } \\
\begin{array}{l}\text { Realizarón cambios en el servicio que } \\
\text { brindan. }\end{array}\end{array}$ & 9 \\
\hline $\begin{array}{l}\text { Realizarón mejoras al establecimiento. } \\
\text { Re }\end{array}$ & 3 \\
\hline
\end{tabular}

\section{Problemas económicos:}

Unos no tenian problemas económicos por su cercania con la universidad, pero otros no sacaban las ventas diarias que esperaban.

\section{Personal suficiente:}

Todos los negocios indicarón que contaban con pocos empleados pero suficientes para cubrir las necesidades de los clientes, que lo importante es que son trabajadores.

6.Cambios de área que el personal podia sufrir:

Varios establecimientos indicarón que no era bueno que los estuvieran cambiado de área, debido a que cada quien sabía lo que tenían que hacer, en cambio en otras área tenían que empezar a trabajar sin conocimientos previos.

7.Cómo consideran el impacto que tiene su publicidad:

\begin{tabular}{|l|r|}
\hline Alto & 4 \\
\hline Bajo & 3 \\
\hline Medio & 13 \\
\hline
\end{tabular}

8.Por que consideran que los clientes eligen su establecimiento:

\begin{tabular}{|l|r|}
\hline Servicio & 6 \\
\hline Producto & 6 \\
\hline Comodidad & 8 \\
\hline
\end{tabular}

\section{Su ubicación es importante:}

Para algunos su ubicación no es suficientemente visible para los clientes considerán que la ubicación es importante y los que no lo consideran importante es porque su establecimiento es cerca de la universidad o escuela y ésto les genera mayores utiidades.

10.El tipo de clientela que consumen en el establecimiento:

\begin{tabular}{|l|r|}
\hline Estudiantes & 16 \\
\hline Civil & 3 \\
\hline
\end{tabular}


Otra (trabajadores, choferes)

\section{Conclusiones}

En los resultados de las encustas se puede observar que los negocios no generan la mayoría de sus cambios, sin embargo, tratan de adaptarse a ellos para no cerrar lo que es bueno para mantenerse en el mercado y seguir generando empleos.

En lo que corresponde desde el punto de vista de la administración los empleados consideran que los dueños visión hacia donde quierenllegar, pero carecen de un adecuado control de gastos.Para ello es necesario llevar un registro diario de entradas y salidas de dinero, para poder hacer inversiones que valgan la pena y que permita atraer más clientes.

Todo personal es necesario que cumpla con sus funciones, pero en este tipo de negocios que son muyb pequeños, hay que aprender de todo para cuando un empleado no pueda asistir, otro pueda hacer su trabajo, con el objetivo de que el negocio siga funcionando.

Este tipo de negocios constantemente debe establecer estrategias para identificar los motivos por los cuáles los clientes prefieren el servicio o cómo se puede mejorar.

La ubicación tiene que ser visible a la población o en su caso establecer estrategias de publicidad.

El municipio de Tlahuelilpan debe considerar que cuenta con una economía activa en lo que corresponde a los negocios de comida rápida, debido a la llegada de la universidad, a los centros educativos con los que cuenta el municipio y la plaza de los días martes que generan un estado económico para quienes tienen este tipo de micronegocio.

\section{Referencias}

ACADEM Escuela Latinoamericana De Management Y Marketing David Polo Moya. (7 de noviembre de 2017). El Ciclo de Administración. Obtenido de El
Ciclo de Administración:

https://www.100negocios.com/administracioncomo-opera-un-negocio

Arreguin, S. D. (2016). La microempresa en desarrollo. Obtenido de La microempresa en desarrollo: https://www.redalyc.org/

CESAR, M. (11 de MARZO de 2019). EL SOL HIDALGO. Obtenido de EL SOL HIDALGO:

https://www.elsoldehidalgo.com.mx/local/regional /tlahuelilpan-busca-activar-su-economia4139131.html

Figueroa, W. V. (2017). Estrategias de Marketing para la mejora de un servicio de comidas rápidas. Obtenido de Estrategias de Marketing para la mejora de un servicio de comidas rápidas.

Infestareis, Organización internacional del trabajo. (2018). El poder de lo pequeño: hay que activar la potencia económica. Obtenido de El poder de lo pequeño: hay que activar la potencia económica : http//:El-poder-de-lo-pequeño:-ha- que-activar-lapotencia-económica.com

Redacción Portafoio. (2018). Mercado de comida rápidas con potencialde explotar. Obtenido de COMIDA RAPIDA: WWW.PORTAFOLIO.COM

Rene Rivera, N. L. (2016). Políticas de Apoyo a la Microempresa Informal. Obtenido de Microempresas Pequeñas Medianas y El poder de lo Pequeño:

https://scholar.google.es/scholar?hl=es\&as_sdt=0 $\% 2 \mathrm{C} 5 \& \mathrm{q}=$ potencial+de+las+microempresas+en+

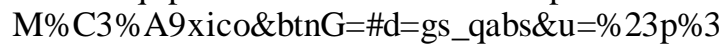
DvP2yGBqv2X8J

Segarra, A. y. (14 de 1 de 2002). New firms' survival and market turbulence:., 20(1), 1-14. Spain: New evidence from Spain. Obtenido de Review of Industrial Organization. 